\title{
Efficacité des pièges à paraphéromone dans la protection des vergers de manguiers greffés contre les mouches des fruits au Togo
}

\author{
Atti TCHABI ${ }^{*}$, Abouwaliou Nafadjara NADIO ${ }^{1}$, Bonoukpoè Mawuko SOKAME ${ }^{4}$ \\ Magnim Essolakina BOKOBANA ${ }^{1}$, Mondjonnesso GOMINA³ ${ }^{3}$, Agbéko Kodjo TOUNOU² \\ et Komla SANDA ${ }^{1}$
}

\author{
${ }^{1}$ Laboratoire du Centre d'Excellence en Protection Durable des Cultures, Institut Supérieur des Métiers de \\ l'Agriculture (ISMA), Université de Kara, BP. 404 Kara-Togo. \\ ${ }^{2}$ Laboratoire de Recherche sur les Agroressources et la Santé Environnementale, Ecole Supérieure \\ d'Agronomie, Université de Lomé, B.P. 1515 Lomé, Togo. \\ ${ }^{3}$ Laboratoire de Biologie Animale et de Zoologie, Faculté des Sciences, Université de Lomé, B.P. 1515 Lomé, \\ Togo. \\ ${ }^{4}$ International Centre of Insect Physiology and Ecology (ICIPE), P.O. Box 30772-00100, Nairobi, Kenya. \\ *Auteur correspondant ; E-mail : attitchabi@yahoo.fr
}

Received: 26-10-2020

Accepted: 26-02-2021

Published: 28-02-2021

\section{RESUME}

Les mouches de fruits en infestant les mangues greffées causent des pertes considérables et un manque à gagner aux différents acteurs en passant par les producteurs jusqu'aux consommateurs. Pour venir à bout de ces insectes nuisibles, la pulvérisation des insecticides chimiques de synthèse est généralement la solution la plus commune et de premier recours dans toutes les régions du Togo. Mais cette méthode reste dangereuse pour la santé humaine et environnementale et peu rentable économiquement. Cette étude a pour objective de tester l'efficacité du piège Biofeed, une solution alternative, respectueuse de l'environnement et de la santé de l'Homme. Trois vergers d'environ 1 ha pour les trois traitements (piège Biofeed, piège M3 et le control) ont été choisis dans chacune des cinq régions du Togo. Les pièges ont été installés à la prématuration des fruits à une densité de 25 pièges/ha et sont suivis toutes les deux semaines jusqu'à la récolte finale en procédant aux échantillonnages des mangues pour l'évaluation du taux infestation et identification des espèces de mouche de fruits. Après la pose et le suivi des infestations, les pièges Biofeed se sont révélés efficaces en réduisant sur le plan national les dégâts des mouches de fruits de 73,60\% tandis que les pièges M3 n'ont entrainé qu'une réduction de 39,94\%. Les vergers ayant bénéficiés de ces pièges ont connu des taux d'infestations faibles promettant une bonne récolte. L'incubation des mangues récoltées dans les vergers ont permis de recenser 4 genres de mouches de fruits (Bactrocera, Ceratitis, Dacus, Zeugodacus) et Bactrocera dorsalis est l'espèce la plus abondante retrouvée sur ce fruit d'importance économique.

(C) 2021 International Formulae Group. All rights reserved.

Mots clés : Mouches de fruit, mangue greffée, piège Biofeed, piège M3. 


\title{
Efficacy of parapheromone traps in the protection of grafted mango orchards against fruit flies in Togo
}

\begin{abstract}
Fruit flies by infesting grafted mangoes cause considerable losses and loss of profit to the various actors range from producers to consumers. To control these pests, synthetic chemical insecticides use is generally the most common and first resort solution in all regions of Togo. But this method remains dangerous for human and environmental health. The objective of this study was to test the effecacy of Biofeed trap, an alternative solution that is environmentally friendly in comparison with a trap already used in the field (M3 traps based on food bait). Three orchards of about 1 ha for the 3 treatments (Biofeed trap, M3 trap and control) were chosen in each of the five regions of Togo. The traps were installed at the premature fruit maturity at a density of 25 traps / ha and were monitored every two weeks until the final harvest by sampling the mangoes for the assessment of the infestation rate and identification of fruits fly species. After setting traps and monitoring the infestations, Biofeed traps proved effective by reducing fruit fly damage by $73.60 \%$ nationally, while M3 traps by $39.94 \%$. The orchards that benefited from these traps had low infestation rates that promissing a good harvest. Incubation of the mangoes harvested in the orchards have allowed to identify 4 genera of fruit flies (Bactrocera, Ceratitis, Dacus, Zeugodacus) and Bactrocera dorsalis is the most abundant species found on this economically important fruit.
\end{abstract}

(C) 2021 International Formulae Group. All rights reserved.

Keywords: Fruit flies, grafted mango, Biofeed and M3 traps.

\section{INTRODUCTION}

La filière mangue constitue une composante essentielle du secteur horticole dans beaucoup de pays de la Communauté Economique des Etats de l'Afrique de l'Ouest (CEDEAO). Elle revêt dans l'Afrique de l'Ouest une importance particulière dans la diversification de revenus, la création de richesses, la contribution à la sécurité alimentaire et nutritionnelle, au développement économique et sociale (HLPE, 2016). Elle constitue une bonne opportunité de revenu pour les petits agriculteurs en général. Cependant, depuis plusieurs années, cette filière est confrontée à la problématique de la mouche des fruits. L'introduction et la dispersion de nouvelles espèces invasives d'origine asiatique dans toute l'Afrique de l'Ouest ont accentué considérablement l'importance des dégâts sur les mangues au niveau régional. Plus de 40 espèces endémiques de mouches phytophages ont été inventoriées en Afrique de l'Ouest, et sont responsables de destructions pouvant atteindre jusqu'à $100 \%$ des vergers dans certaines zones (Dieng et al., 2019). Les mouches des fruits étant classées « insectes de quarantaine », aucun fruit piqué renfermant une larve ne peut être exporté sous peine du rejet et de la destruction totale du lot de mangues par les services phytosanitaires européens (CTA, 2012). Les conséquences de ces interceptions et destructions de mangues de l'espace CEDEAO arrivant sur le marché européen sont l'abandon et la destruction des vergers de manguiers au profit d'autres cultures (Rapport ProMangue, 2019).

Ainsi, afin d'apporter une contribution dans la lutte contre ces insectes nuisibles, une étude a été réalisée afin d'évaluer l'efficacité des pièges à paraphéromones de technologie Biofeed dans la protection des vergers de manguiers greffés contre les attaques des mouches des fruits (Tephritidae frugivore). Avec l'utilisation abusive des produits phytosanitaires dans les vergers, ce travail de recherche a pour finalité de proposer une alternative de lutte efficace et écologiquement fiable dans les vergers du Togo.

\section{MATERIEL ET METHODES \\ Choix des sites}

Des vergers de manguiers greffés ont été choisis dans les cinq régions économiques 
du Togo (maritime, plateaux, centrale, Kara et savanes) pour réaliser les expérimentations afin d'avoir des données à dimension nationale. Au total, quinze (15) vergers ont été choisis (3 par régions) dont 10 vergers ont été piégés ( 2 par régions) avec un verger par région comme témoin absolu. Les vergers ont été sélectionnés dans les localités suivantes: Badja dans la région maritime, Atakpamé dans la région des plateaux, Sokodé dans la région centrale, Kantè dans la région de la Kara et Dapaong dans la région des savanes. Les critères retenus pour le choix des vergers sont l'accessibilité, l'homogénéité de variété de manguier dans le verger, la superficie des vergers d'au moins 2 ha, l'âge des vergers (cinq (5) à dix (10) ans) et l'absence de tout traitement chimique dans les vergers et à proximité durant les expérimentations.

\section{Matériels techniques}

Il est constitué des pièges à paraphéromones de technologie Biofeed (pièges Biofeed) dont le principe actif est le spinosad $(0,035 \mathrm{~g} /$ piège) (Photo 1A). Ce composé est une matière active d'origine naturelle qui présente un effet insecticide (neurotoxique). Le piège à paraphéromone de la technologie Biofeed consiste en un dispositif à écran plat contenant une substance liquide. Ce matériel utilisé dans les vergers de la vigne en Europe se teste pour la première fois dans les vergers de manguiers en Afrique. La mouche de fruits, attirée par ce dispositif, vient se poser sur le panneau pour s'alimenter et meurt peu de temps après. Le dispositif d'alimentation agit comme une station d'alimentation à long terme utilisant un mécanisme qui assure une libération lente du liquide de qualité constante sur une longue période, allant jusqu'à 6 mois. Dans l'optique de mettre en exergue l'efficacité de ce paraphéromones, des pièges $\mathrm{M} 3$ reconnus comme pièges de référence ont été choisis comme témoin relatif. Les pièges M3 sont composés d'appâts alimentaires utilisés depuis quelques années dans les vergers contre les mouches de fruits par la Direction de la Protection des Végétaux (DPV) au Togo (Photo 1B).

\section{Mise en place des pièges}

Concernant la méthodologie, un périmètre de deux (2) ha de verger en un seul tenant choisi est divisé en deux parties égales. Sur la première partie sont placés les pièges Biofeed et sur la partie restante, les pièges de référence (piège M3) devant servir de contrôle positif. Les pièges étaient suspendus à une branche principale du tiers inférieur $\mathrm{du}$ feuillage, à une hauteur moyenne de 1,5 à 1,8 $\mathrm{m}$ au-dessus du sol et à l'abri de la lumière du soleil pour éviter la dégradation rapide de la matière active des pièges. La branche supportant le piège a été préalablement enduite de graisse afin de prévenir toute activité prédatrice des fourmis contre les mouches adultes mortes capturées dans le piège. Sur chaque parcelle de 1 hectare, cinq pièges, ont été placés en ligne à une distance minimale de $20 \mathrm{~m}$ entre eux. Le système a été répété cinq fois, soit un total de 25 pièges / ha. Dans la même localité, à environ un (1) kilomètre, un verger de 1 ha sans piège et non traité a servi de témoin absolu. Compte tenu de la sensibilité des variétés de mangue, les vergers comportant les mêmes variétés ont été choisis. Les essais ont été conduits sur deux saisons successives de production de mangues (2018 et 2019), chacun de la période de la pré maturation à la récolte des mangues.

\section{Efficacité des pièges sur le taux d'infestation dans les vergers}

Le suivi des infestions des mouches sur les mangues s'est fait toutes les deux semaines de la pré maturation jusqu'à la récolte finale. Dans chaque verger, le choix des manguiers s'est fait de façon aléatoire en suivant pour chaque parcelle le parcours «W». Cinq (5) manguiers ont été choisis par verger et dix fruits sont cueillis au hasard. Les dix mangues cueillies sur un manguier constituent un lot représentant une répétition. En définitive, cinq lots (5 répétitions) de dix mangues sont constitués par verger.

Pour déterminer la présence ou l'absence de piqûres des mouches des fruits, une observation visuelle à l'œil nu et/ou à la loupe à main est faite des cinquante mangues individuellement. Toute trace de piqûre est 
prise en compte. Une deuxième observation complémentaire a consisté à peler sur-lechamp les mangues et procéder à des observations directes à l'intérieur des fruits. Les observations internes sont visuelles et les zones d'infestation sont examinées à l'aide d'une loupe à main pour rechercher d'éventuels œufs et/ou larves. Une mangue est considérée comme infestée lorsqu'elle présente au moins une piqûre d'une mouche des fruits et /ou présente d'éventuels œufs et/ou larves. Les taux d'infestation des vergers ont été calculés en rapportant le nombre de mangues présentant au moins une piqûre des mouches des fruits par rapport au nombre total de mangues inspectées.

$T=\frac{N P i q}{N T I} \times 100$

\section{$\mathrm{T}$ : Taux d'infestation en pourcentage}

NPiq: nombre de mangues présentant au moins une piqûre des mouches des fruits et /ou présente d'éventuels œufs et/ou larves

NTI : nombre total de mangues inspectées.

\section{Identification des différentes espèces de mouches par incubation des mangues échantillonnées au laboratoire}

L'objectif de l'incubation était d'identifier les espèces de ces ravageurs associés à la mangue dans les vergers. Elle a également permis de récupérer et d'identifier les espèces de parasitoïdes associées aux mouches des fruits sur les mangues dans les différents vergers prospectés.

A chaque observation, cinq mangues ont été cueillies sur les cinq manguiers choisis au hasard. Les mangues cueillies dans chaque verger ont été ramenées au laboratoire et incubées séparément par lot de dix (Photo 2). Les mangues de chaque lot ont été incubées sur du support grillagé dans des bassines plastiques contenant du sable humidifié et stérilisé. Les nymphes émergées ont été recueillies du sable par tamisage toutes les semaines. Elles ont été comptées, transférées dans des cages d'élevage et suivies jusqu'à émergence des adultes de mouches ou d'éventuels parasitoïdes.

L'identification des espèces de mouches des fruits et de parasitoïdes après émergence ont été faite au département de Zoologie et de Biologie Animale de la Faculté des Sciences de l'Université de Lomé.

\section{Analyses des données}

L'analyse statistique des taux d'infestation des vergers a été réalisée à l'aide du logiciel XLSTAT 7.5.2. Le test de Duncan au seuil de $5 \%$ a permis de discriminer les groupes homogènes des moyennes des différentes données. Les histogrammes illustrant les taux d'infestation par zone d'étude ont été faits à l'aide du tableur Excel.

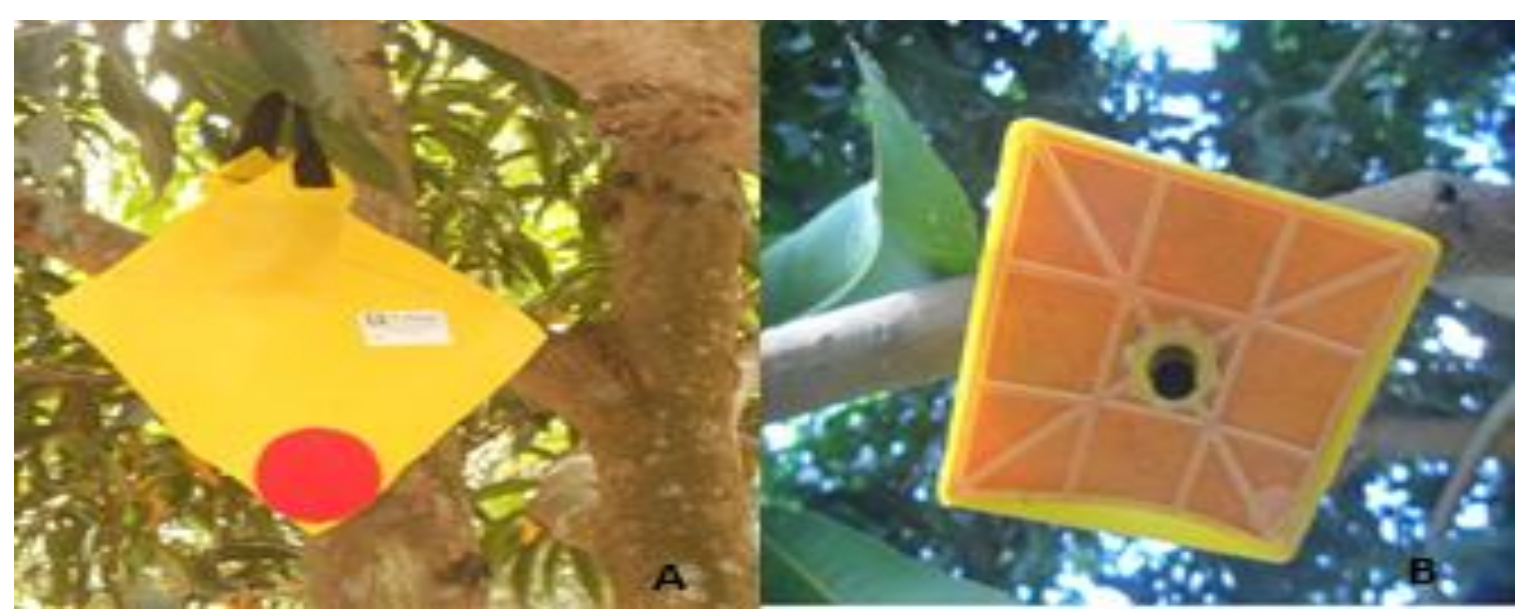

Photo 1 : A- pièges Biofeed et B- Pièges M3. 


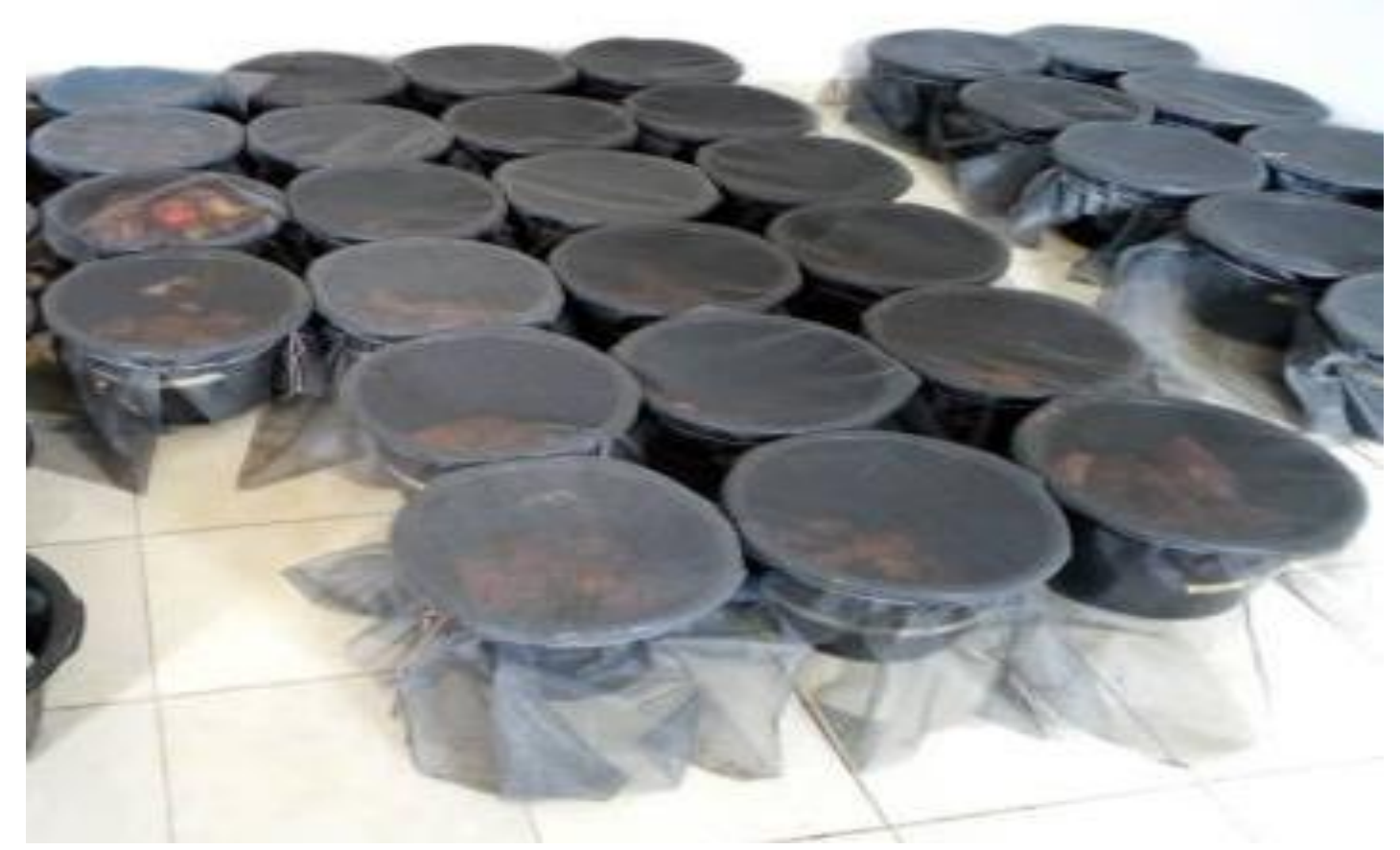

Photo 2: Des mangues dans des bassines plastiques contenant du sable humidifié et stérilisé permettant de recueillir les nymphes des espèces de mouches de fruits émergées.

\section{RESULTATS}

\section{Effet des pièges à paraphéromone sur le} taux d'infestation des mouches de fruits

Dans la région des Savanes, les vergers protégés par les pièges Biofeed et $\mathrm{M} 3$ ont connu des taux d'infestations respectifs de 5 et $15 \%$ contre un taux de $76 \%$ dans le verger témoin (Figure 1). Les pièges Biofeed et M3 ont réduit respectivement les infestations de 93,42 et $80,26 \%$. A Kantè dans la région de la Kara, les taux d'infestation sont de 7,5\% pour les vergers protégés par les pièges Biofeed et 16,6\% dans les vergers piégés à M3 (Figure 1). Les infestations ont été significativement plus importantes dans le verger témoin absolu où l'on a enregistré un taux de $30 \%$. Les réductions des dégâts sur les manguiers greffés sont respectivement de 75 et $44,66 \%$ pour les pièges Biofeed et M3. Dans la région centrale, le taux d'infestation moyen a été de $53 \%$ dans les vergers témoins tandis que dans les vergers piégés avec les appâts Biofeed et M3, les taux ont été respectivement de 4,12 et 7,5\%. Par rapport aux témoins, les pièges Biofeed et M3 ont réduit respectivement le taux d'infestation de ces mouches de fruits de 92,23 et $85,85 \%$. Dans la région des plateaux, les taux d'infestation ont été de $13,33 \%$ pour les vergers à pièges Biofeed, $40 \%$ pour les vergers à pièges $\mathrm{M} 3$ et $24 \%$ dans les vergers témoins. Dans la région Maritime, l'évaluation des dégâts dans ces vergers a révélé que les taux d'infestations sont de $80 \%$ dans les vergers à pièges $\mathrm{M} 3$ et de $40 \%$ dans les vergers à pièges Biofeed tandis que le témoin est à $82 \%$ de dégâts (Figure 1). Les pièges Biofeed et M3 ont donc réduit respectivement de 40 et $2,44 \%$ les attaques de ces mouches de fruits.

En faisant la moyenne de ces résultats des cinq zones d'études, les taux moyens d'infestation sont de $13,99 \pm 2,33 \%$ dans les vergers à pièges Biofeed, $31,83 \pm 3,58 \%$ dans les vergers à pièges $\mathrm{M} 3$ et $53 \pm 4,2 \%$ dans les vergers témoins (Figure 2). L'analyse de variance des taux moyens d'infestations des vergers protégés aux pièges Biofeed, M3 et des vergers témoins absolus a montré une différence significative au test de Duncan au 
seuil de 5\% ( $\mathrm{F}=101,88 ; \mathrm{ddl}=4 ; 10, \mathrm{p}=0,0015$ $<0,05)$. Par rapport aux témoins absolus, les taux de réduction moyens des attaques des mouches de fruits sont $73,60 \%$ pour les pièges Biofeed et $39,94 \%$ pour les pièges M3. Lorsqu'on se réfère aux taux d'infestations par région des vergers à pièges Biofeed, les vergers de la région Centrale ont été moins attaqués avec un taux d'infestation de $4,12 \%$ suivis de ceux de la région de la Savane (5\%), de la région Kara $(7,5 \%)$, des plateaux $(13,33 \%)$ et Maritime (40\%).

L'observation de l'état sanitaire des mangues après dissection a montré également des fruits sains et non piqués dans vergers à pièges Biofeed (Photos 3). Cependant l'on rencontre beaucoup de fruits pourris ou tombés dans les vergers témoins et quelques-uns dans les vergers piégés à M3.

\section{Détermination des différentes espèces de} mouches de fruits et leurs parasitoïdes associés après incubation des mangues greffées

L'incubation des lots de mangues ramenés au laboratoire a permis d'évaluer la richesse spécifique des espèces de mouches de fruits après émergence des adultes. Cette étude a révélé la présence de quatre importants genres (Bactrocera, Ceratitis, Dacus, Zeugodacus) (Tableau 1). Bactrocera dorsalis a été l'espèce la plus abondante.

$\mathrm{Au}$ total, 617 individus des hyménoptères parasitoïdes indigènes associés à 3275 individus de mouches des fruits (toutes espèces confondues) ont été récupérés au cours de l'incubation des fruits en provenance des différentes zones d'étude. Ces parasitoïdes sont associés à 4 espèces de mouches des fruits à savoir Bactrocera dorsalis, Ceratitis capitata, Ceratitis colae et Ceratitis cosyra. Ils appartiennent à 6 espèces, 4 genres et 2 familles. La famille des Braconidae est la plus représentée avec 99,35\% (613 individus) de l'effectif total. Dans cette famille, Fopius caudatus Szépliget est le plus abondant avec 605 individus (soit 98,06\%). Diachasmimorpha fullawayi Silvestri, Fopius sp., Psyttalia sp. et Bracon sp. représentent respectivement $0,49 \%$ (3 individus), $0,32 \%$ (2 individus), $0,32 \%$ ( 2 individus) et $0,16 \%$ (1 individu) (Tableau 2). La famille des Eulophidae est représentée par la seule espèce indéterminée avec $0,65 \%$ (4 individus).

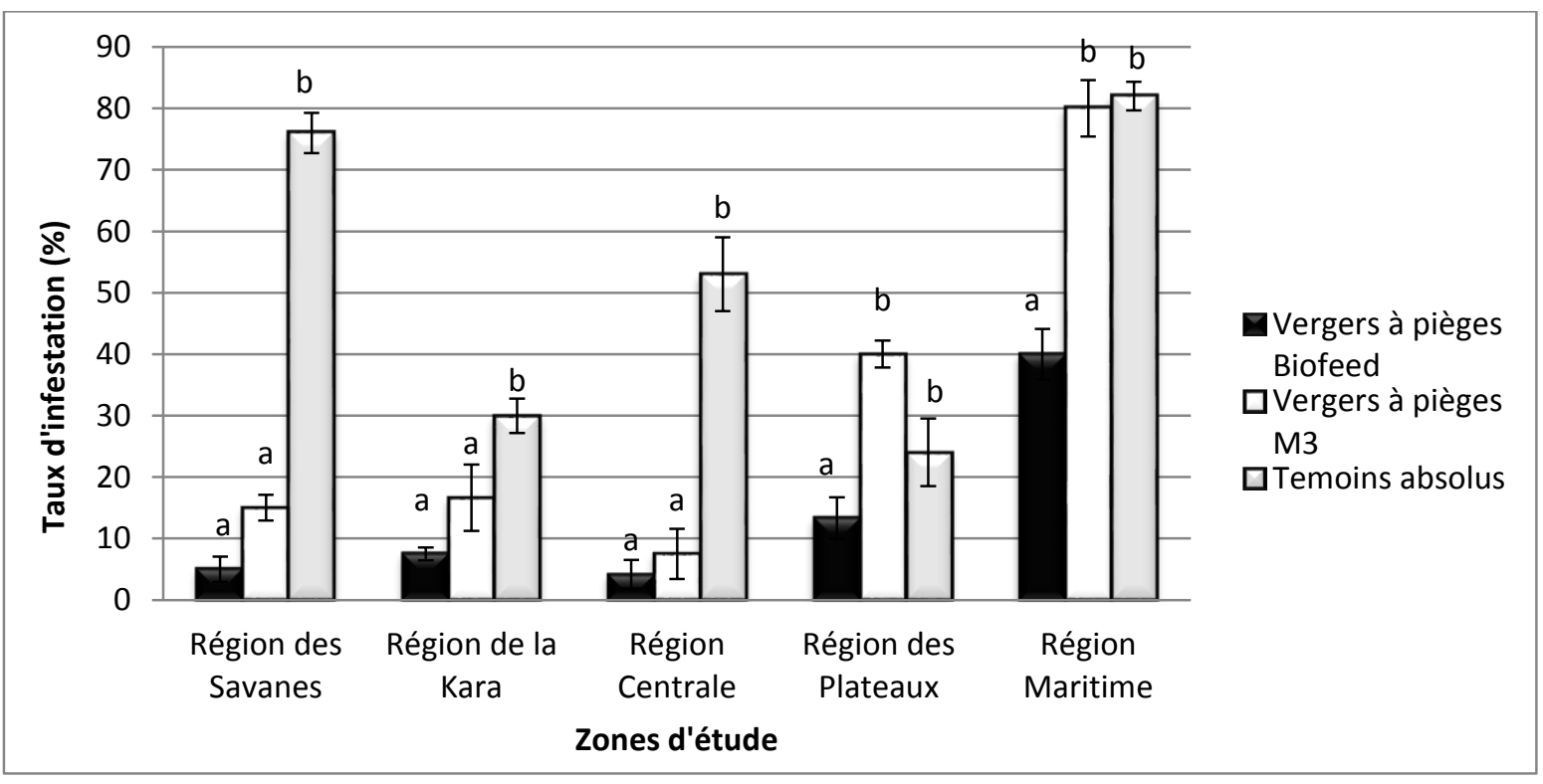

Figure 1 : Taux moyens d'infestations des vergers par zones d'étude.

Les barres portant de différentes lettres sont significativement différentes (test de Duncan au seuil de 5\%). 


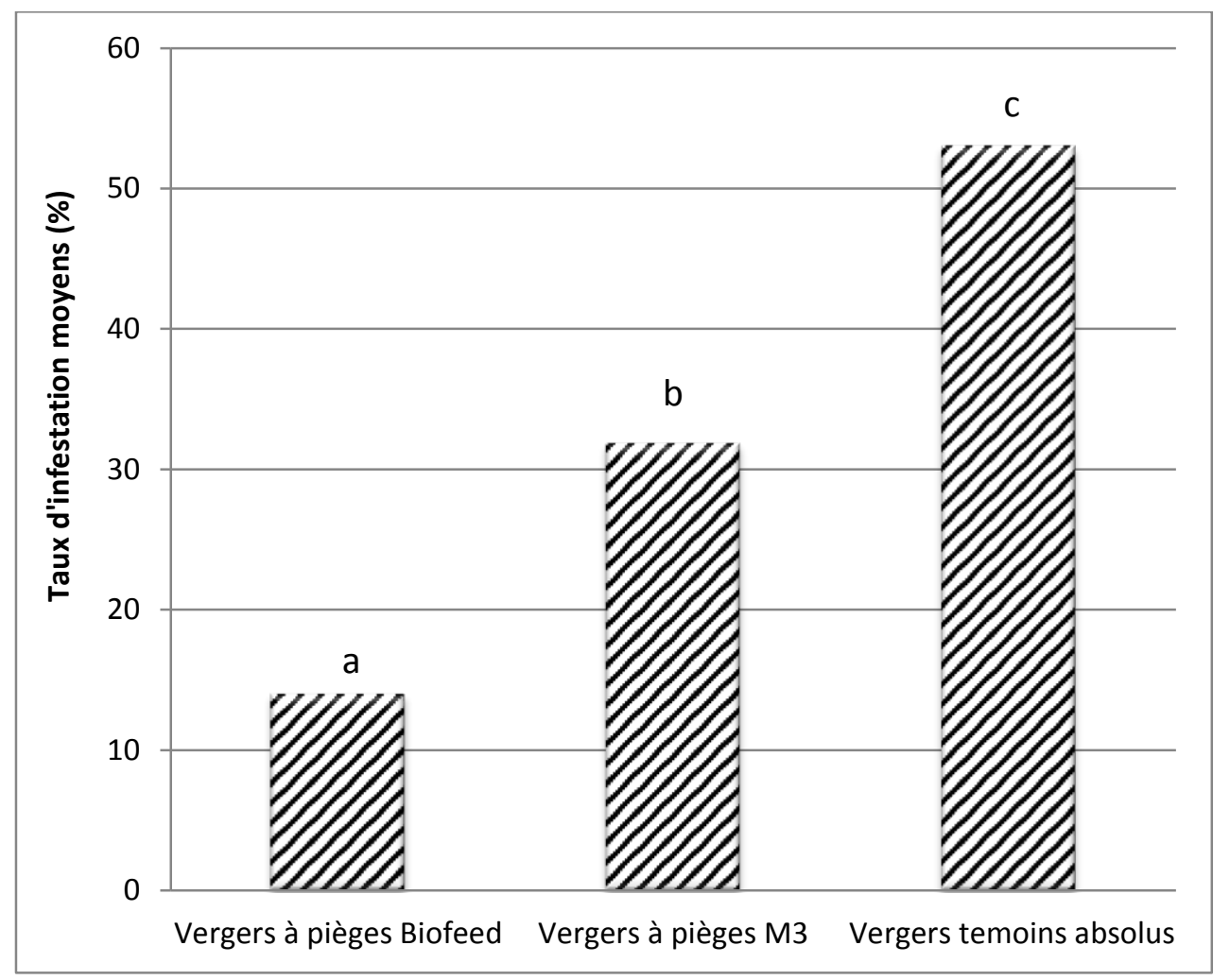

Figure 2 : Taux moyens d'infestations des vergers en fonction des différents traitements. Les barres portant de différentes lettres sont significativement différentes (test de Duncan au seuil de 5\%).
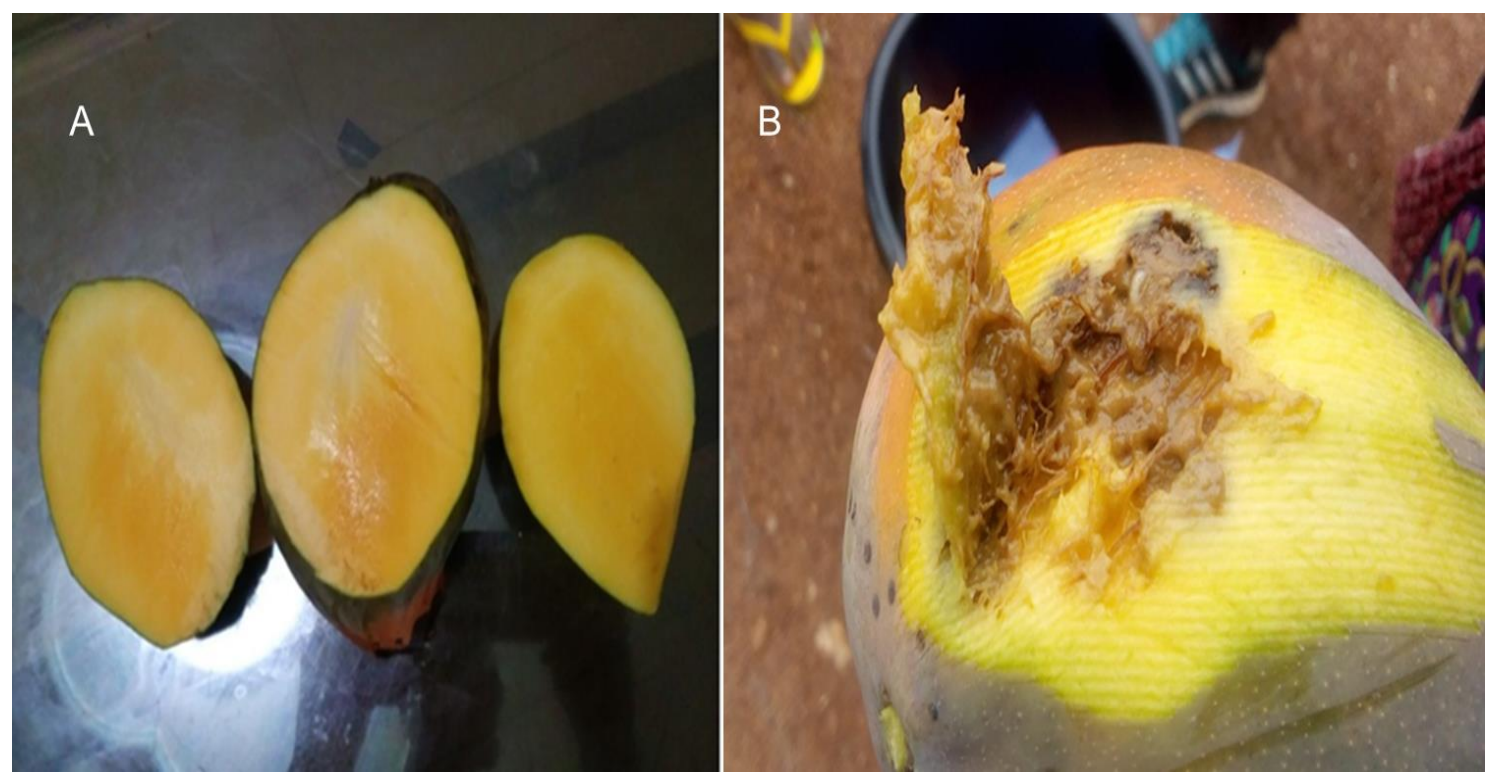

Photo 3 : Coupes montrant la pulpe saine des mangues non attaquées (A) et la pulpe de mangue attaquée présentant une pourriture (B). 
Tableau 1 : Les espèces de mouche de fruit inventoriées selon les zones d'étude.

\begin{tabular}{|c|c|c|c|c|c|}
\hline Espèces & $\begin{array}{c}\text { Région } \\
\text { maritime }\end{array}$ & $\begin{array}{c}\text { Région des } \\
\text { plateaux }\end{array}$ & $\begin{array}{c}\text { Région } \\
\text { centrale }\end{array}$ & $\begin{array}{c}\text { Région de } \\
\text { la Kara }\end{array}$ & $\begin{array}{c}\text { Région des } \\
\text { savanes }\end{array}$ \\
\hline Bactrocera dorsalis (Hendel) & + & + & + & + & + \\
\hline Ceratitis anonae Graham & & + & + & & \\
\hline Ceratitis bremii Guérin-Méneville & & + & + & + & + \\
\hline Ceratitis capitata (Wiedemann) & + & & + & + & \\
\hline Ceratitis cosyra (Walker) & + & + & + & + & + \\
\hline Dacus bivittatus (Bigot) & + & + & & & \\
\hline Dacus diastatus Munro & + & & + & & \\
\hline Dacus humeralis (Bezzi) & + & + & + & + & + \\
\hline Dacus langi Curran & & + & & + & \\
\hline Dacus punctatifrons Karsch & + & & + & & \\
\hline Zeugodacus cucurbitae (Coquillett) & + & & & & \\
\hline Richesse Spécifique & 8 & 7 & 8 & 6 & 4 \\
\hline
\end{tabular}

Tableau 2 : Proportions des individus des différentes espèces de parasitoïdes émergés des fruits incubés au laboratoire.

\begin{tabular}{clccc}
\hline Familles & Espèce & Hosts & Effectif & Pourcentage \\
\hline & Bracon sp. $^{1}$ & Bd & 1 & 0,16 \\
& Diachasmimorpha. Fullawayi $^{1,2}$ & Bd,Cca & 3 & 0,49 \\
Braconidae & Fopius caudatus $^{3}$ & Bd, Cca, Cco, & 605 & 98,06 \\
& ${\text { Fopius } \text { sp. }^{4}}^{*}$ Psyttalia sp. $^{5}$ & Ccss & 2 & 0,32 \\
\hline \multirow{2}{*}{ Eulophidae } & Eulophidae $^{* 1}$ & Bd & 2 & 0,32 \\
\hline Total & & Cca & 4 & 0,65 \\
\hline
\end{tabular}

*Espèce de parasitoïde appartenant à la famille des Eulophidae non identifié. ${ }^{1}$ site de région maritime, ${ }^{2}$ site de région Kara, ${ }^{3}$ sites des 5 régions, ${ }^{4}$ site de région plateaux, 5 site région centrale. Bd=Bactrocera dorsalis, Cca=Ceratitis capitata, $\mathrm{Cco}=$ Ceratitis colae et $\mathrm{Ccos}=$ Ceratitis cosyra . 


\section{DISCUSSION}

Au regard de ces résultats dans les cinq régions d'étude, les vergers ayant des pièges Biofeed et M3, excepté le verger M3 dans la région maritime, ont connu tous des taux d'infestation faibles par rapport aux vergers témoins absolus. Ces pièges ont réduit les attaques des mouches de fruits dans ces vergers, ce qui permettra aux producteurs de faire de bonnes récoltes. Ils ont été efficaces dans la protection de ces vergers en réduisant les piqûres des ravageurs sur les mangues greffées. Le principe actif spinosad contenu dans les pièges Biofeed nourrit beaucoup d'espoir depuis quelques années dans la lutte contre les mouches de fruits. Spinosad agit très rapidement après contact ou ingestion par l'insecte. Son action par ingestion est jugée de 5 à 10 fois plus efficace. Ses effets sont rapides, ce qui est relativement inhabituel pour un produit issu de fermentation naturelle. Une fois absorbé par l'insecte, le spinosad atteint rapidement le système nerveux central et provoque une paralysie totale du ravageur qui ne peut plus s'alimenter. Des essais ont été mis en place en Europe, sur de grandes cultures telles vignes, olivier ou agrumes. Les bons résultats obtenus sur cératite ou la mouche de l'olive, sur de grandes surfaces avec une formulation adulticide ont été encourageants (GRAB, 2002).

Dans la région des Plateaux, la totalité des vergers a connu une faible infestation. $\mathrm{Ce}$ faible taux d'infestation des vergers témoins pourrait être dû aux conditions climatiques défavorables dans cette région qui n'ont pas permis la pullulation de ces ravageurs. Le fort taux d'infestation observé dans les vergers piégés à $\mathrm{M} 3$ comparativement au témoin dans cette région pourrait s'expliquer par l'état défaillant de ces pièges dont les appâts alimentaires ont été attaqués par les moisissures. Dans les cinq régions de l'étude, bien que les deux pièges aient réduit les taux d'infestation dans ces vergers, les pièges Biofeed ont été plus efficaces et stables dans la réduction des dégâts de ces mouches de fruits. Par ailleurs, les résultats de l'étude ont montré que les plus faibles taux d'infestation ont été obtenus dans la région centrale alors que les taux les plus élevés ont été obtenus dans la région maritime. Cette faible infestation dans la région Centrale pourrait s'expliquer par le fait que la plupart des vergers était bien entretenue, le schéma cultural des manguiers est le plus souvent respecté et la plupart des producteurs pense déjà à l'exportation extérieure. Plusieurs vergers de la région Maritime (Zone la plus infestée) sont laissés à l'abandon sans suivi. Les producteurs se contentent de venir faire la récolte à la maturation des fruits.

Au total, 11 espèces des mouches des fruits ont été inventoriées sur l'ensemble des sites de l'étude. La plupart de ces espèces avait été déjà identifiée au Togo (Amevien et al., 2009) et dans d'autres régions d'Afrique (Mwatawala et al., 2004 ; Vayssières et al., 2004 et 2005). Batrocera dorsalis a été l'espèce majoritaire. Cette impressionnante présence s'expliquerait par une forte invasion des sites par cette espèce de Tephritidae. Ekesi et al. (2009) et Mwatawala et al. (2009) ont spéculé que la mangue est l'un des fruits préférés de $B$. dorsalis et par conséquent, elle pullule dans les zones productrices de mangues. La faible capture des espèces indigènes serait due au statut de super compétiteur de $B$. dorsalis vis-à-vis de ces espèces mais également du fait de l'existence d'une compétition interspécifique entre les espèces (Duyck et al., 2004).

De plus, cette étude a mis en exergue la présence des parasitoïdes où six espèces ont été inventoriées sur l'ensemble des sites. L'efficacité de la para-phéromone Biofeed dans cette présente étude vient à point nommé pour la préservation de ces espèces de parasitoïdes dans le cadre d'une gestion intégrée et durable des mouches fruits dans les vergers de manguier.

\section{Conclusion}

$\mathrm{Au}$ vu de ces résultats d'évaluation de l'efficacité des pièges, il ressort que les pièges Biofeed à paraphéromones contenant un principe actif naturel pourraient bien être recommandés pour une gestion efficace des mouches de fruits dans les vergers de manguiers greffés. Les pièges M3 ont été également efficaces mais instables et facilement attaquables par les moisissures. Pour une protection accrue des pièges paraphéromones, il faut toutefois mettre un accent particulier sur l'animation des vergers (entretien des vergers, ramassage et mis en 
terre des fruits tombés) pour une meilleure efficacité du produit dans un contexte de gestion intégrée des populations des mouches de fruits au niveau national.

\section{CONFLIT D'INTERÊTS}

Les auteurs déclarent ne pas avoir de conflit d'intérêts.

\section{CONTRIBUTIONS DES AUTEURS}

TA a conçu l'étude, a participé à la collecte des données et à la rédaction du manuscrit. NNA et BEM ont participé à la rédaction du manuscrit, acquisition de données et leur interprétation. SBM, TAK, GM ont participé à la rédaction du manuscrit, à l'analyse statistique ainsi qu'à l'interprétation des résultats. SK a supervisé les travaux et participé à la rédaction du manuscrit. Tous les auteurs ont lu et approuvé le manuscrit final

\section{REMERCIEMENTS}

Les auteurs remercient le PPAAOTOGO et l'Université de Kara pour leurs soutiens matériels et financiers pour la réalisation de cette étude

\section{RÉFÉRENCES}

Amevoin K, Sanbena BB, Nuto Y, Gomina M, De Meyer M, Glitho IA. 2009. Les mouches des fruits (Diptera : Tephritidae) au Togo : inventaire, prévalence et dynamique des populations dans la zone urbaine de Lomé. Int. J. Biol. Chem. Sci., 3(5): $\quad 912-920 . \quad$ DOI: https://doi.org/10.4314/ijbcs.v3i5.51057

CTA. 2012. Comment lutter contre la mouche des mangues. Technical Centre for Agricultural and Rural Cooperation, Sri Lanka, 6p.

Duyck PF, David P, Quilici S. 2004. A review of relationships between interspecific competition and invasions in fruit flies (Diptera: Tephritidae). Ecol. Entomol., 29 : 511-520.

DOI: https://doi.org/10.1111/j.03076946.2004.00638.x

Ekesi S, Billah MK, Nderitu PW, Lux AS, Ivan R. 2009. Evidence for competitive displacement of Ceratitis cosyra by the invasive fruit fly Bactrocera invadens (Diptera: Tephritidae) on mango and mechanisms contribution to the displacement. J. Entomol., 102(3): 98991.

DOI:

https://doi.org/10.1603/029.102.0317

HLPE. 2016. Le développement agricole durable au service de la sécurité alimentaire et de la nutrition: quels rôles pour l'élevage? Rapport du Groupe d'experts de haut niveau sur la sécurité alimentaire et la nutrition du Comité de la sécurité alimentaire mondiale, Rome.

GRAB. 2002. Stratégies de lutte contre les mouches des fruits : rétrospective \& perspectives. GRAB, Togo, $7 \mathrm{p}$.

Mwatawala MW, White IM, Maerere PA, Sekondo FJ, De Meyer M. 2004. A new invasive Bactrocera species (Diptera: Tephritidae) in Tanzania. African Entomol., 12(1): 154-156.

Mwatawala MW, De Meyer M, Makundi RH, Maerere AP. 2009. An Overview of Bactrocera (Diptera: Tephritidae) Invasions and their Speculated Dominancy over Native Fruit Fly Species in Tanzania. J. Entomol., 6(1): 18-27. DOI:

https://doi.org/10.3923/je.2009.18.27

Dieng EO, Ndiaye S, Faye PD, Balayara A, Badji K, Sembéne PM. 2019. New inventory of the diversity and seasonal abundance of Tephritid fruit fly species on mango orchards in Senegal. $J$. Entomol. Zool. Stud., 7(6): 975-986.

Rapport Biofeed. 2019. Rapport du projet d'introduction d'une méthode de lutte durable à base de pièges à paraphéromones contre la mouche de fruits dans les vergers de manguiers greffés au Togo (ProMangue). Rapport Biofeed, ISMA-Université de Kara, 104p.

Vayssières JF, Fatogoma S, Moussa N. 2004. Inventaire des espèces de mouches des fruits (Diptera: Tephritidae) inféodées au manguier au Mali et essai de lutte raisonnée. Fruits, 59(1): 1-14. DOI: https://doi.org/10.1051/fruits:2004001

Vayssières JF, Goergen G, Lokossou O, Dossa P, Akponon C. 2005. A new Bactrocera species in Benin among mango fruit fly (Diptera: Tephritidae) species. Fruits, 60: 371-377. DOI: 10.17660/fruits.2009.820.74. 